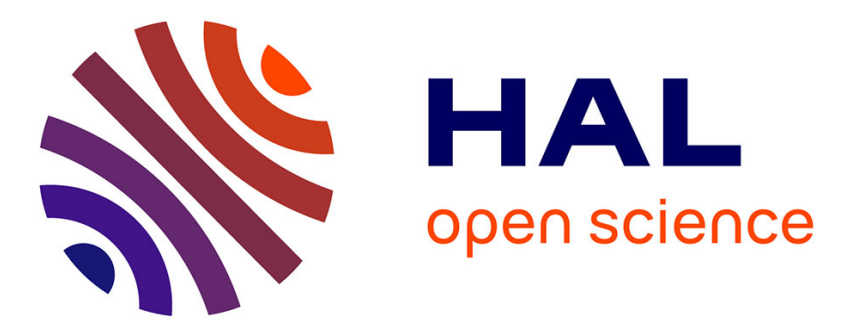

\title{
Trajectory modelling of a planar magnetic cell micropusher.
}

Michaël Dauge, Michaël Gauthier, Emmanuel Piat

\section{To cite this version:}

Michaël Dauge, Michaël Gauthier, Emmanuel Piat. Trajectory modelling of a planar magnetic cell micropusher.. IEEE International Conference on Robotics and Biomimetics, ROBIO'06., Dec 2006, Kunming, China. pp.142-147, 10.1109/ROBIO.2006.340330 . hal-00335145

\section{HAL Id: hal-00335145 \\ https://hal.science/hal-00335145}

Submitted on 28 Oct 2008

HAL is a multi-disciplinary open access archive for the deposit and dissemination of scientific research documents, whether they are published or not. The documents may come from teaching and research institutions in France or abroad, or from public or private research centers.
L'archive ouverte pluridisciplinaire HAL, est destinée au dépôt et à la diffusion de documents scientifiques de niveau recherche, publiés ou non, émanant des établissements d'enseignement et de recherche français ou étrangers, des laboratoires publics ou privés. 


\title{
Trajectory Modelling of a Planar Magnetic Cell Micropusher
}

\author{
Michaël Dauge, Michaël Gauthier and Emmanuel Piat \\ Laboratoire d'Automatique de Besançon - CNRS, ENSMM, UFC \\ 24, rue Alain Savary - 25000 Besançon - France \\ Tel: +33 (0) $381402810-$ Fax: +33 (0) 381402809 \\ Website: http://www.lab.cnrs.fr, E-mail: michael.gauthier@ens2m.fr
}

\begin{abstract}
The improving of the efficiency and the automation of biological cell technologies is a current high stake. One way is to build biological micro-factories which are able to perform a complete biotechnological processes automatically. This technology requires the development of new automatic cell transport system to feed work stations in microfactories. An original magnetic cell micropusher is described in this paper. The ferromagnetic pusher which is submerged in the biological medium follows the movement of a permanent magnet located in the air. This paper focuses on the modelling of the dynamic behaviour of the micropusher in function of the magnet trajectory. The generic model proposed is able to determine pusher trajectory according to the micropusher magnetic properties and the permanent magnet shape and properties. This simulation tool will allow to optimize and to study cell trajectory control in further works.

Index Terms-Biological cell, micromanipulation, mag-
\end{abstract} netic actuation, micropusher.

\section{INTRODUCTION}

Although the In-Vitro Fertilization (IVF) process is usually used by physicians, the success rate stays particularly low. Thus, the improving of this biological process with new biological technologies and new microtechnologies is a high stake. Some microtechnology research groups are working towards a high efficiency IVF process [1-4].

The IVF process whose goal is to fertilize oocytes consists in several manually or teleoperated manipulation steps and requires a high pratical know-how. The major part of the full process time is needed to prepare the oocytes before the technical operations.

Since all steps of the current IVF process are manually made or teleoperated, the design of an automatic IVF microfactory is a solution to improve the ergonomics of current processes and to guarantee a better repeatability of manipulations and injections. In this way, the efficiency of the IVF (around $30 \%$ of pregnancies at the present time in Europe) should be improved.

Furthermore automated manipulations and oocytes treatments will allow to develop other biotechnologies like embryonic stemp cells research for therapeutic cloning. Presently, the objective of the therapeutic cloning is to build organs from one stemp cell. These processes require the culture of a large number of oocytes. In this way, an oocyte treatment microfactory could contribute to therapeutic cloning.

Our microfactory project is presented in figure ??. This bio-microfactory will contain modular microrobots and a cells transport system to move cells inside the microfactory. Each modular microrobot will have a specific function: Oocytes cumulus removal, oocytes properties measurements, fertilization.

The oocytes are placed in the input zone after cumulus removal (see in figure ??). Oocytes go down on the slopping face and stands motionless against the wall. The transfer of oocytes from the input zone to the output area through the processing modules is achieved by a cell micropusher. Some standard modules (test or treatment modules) is placed by the physicians along the cell trajectory. At the end of the process, injected oocytes are placed in the output area where physicians can catch them.

This article presents a magnetic cell transport system for automated IVF micromanipulation. Static behaviour of the micropusher was previously presented in [5] and this article focuses on the description of its dynamic behaviour.

The following section deals with the description of the oocyte transport system and on open-loop oocyte micromanipulations. The third section focused on the dynamic behaviour modelling. Experimental trajectories and comparisons with the model are described in the last section.

\section{Oocyte Transport System}

The aim of our oocyte transport system is to enable cells transport in a channel through the different work stations. The channel volume is filled with a biological medium (see in figure ??). To move our actuator, we chose to use magnetic energy which is a suitable solution for micro-actuation [6-9]. Moreover static magnetic field has no effect on living cells unlike laser trapping system [10].

The original single cell transport system consists in a wireless micropusher which pushes single cell without contact with the external medium. When the cells manipulated are oocytes, the micropusher is a ferromagnetic particle $\left(240 \times 240 \times 25 \mu^{3}\right)$ made in electroplated nickel[11] and actuated with an external magnetic field. In our case external magnetic field is induced by a cylindrical magnet $(\mathrm{NdFeB}, \phi 1.6 \mathrm{~mm}, \mathrm{~h}=0.74 \mathrm{~mm})$ placed on the other side of a vertical wall. The pusher is a passive element which does not require energy stock or electrical connection. In fact, micropusher trajectory is controlled with the magnet movements. Contrary to microfluidic transfer [12], our proposed micropusher is able to push each cell individually. However, at the present only one micropusher can be actuated and multi-pushers control is not considered. In the future, micropusher will be encapsulated because nickel is embryotoxic. The micropusher and cells positions are measured through an optical microscope and a CCD camera. The displacements are made by two motorizes micro-translation stages $\left(\mathrm{PI}^{\circledR}, \mathrm{M}\right.$ 111.1) controlled by a computer. 


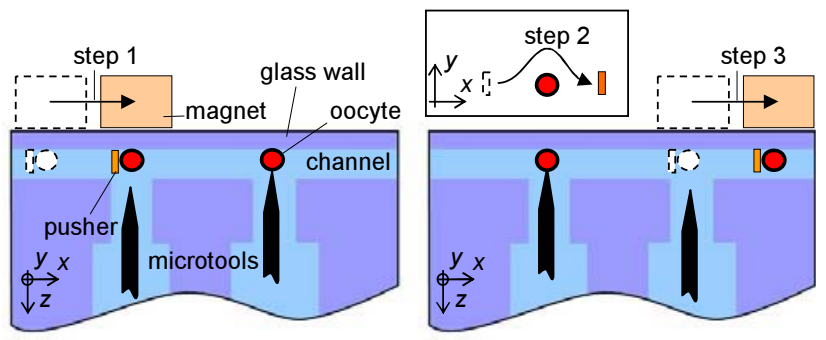

Fig. 1. Example of Cells Treatment Micromanipulation Strategy: Micropusher places an oocyte in front of the first second tool operation is finished, the micropusher goes over the first oocyte (step 2 ) and evacuates the second oocyte (step 3).

The figure 1 describes an example of some micropusher trajectories used to position oocytes. It's a linear view of the in vitro fertilization microfactory presented in figure ??. The first degree of freedom (DOF) along $x$ axis is used to push the micro-object. The second $D O F$ along $y$ axis is used to go over the micro-object. Micropusher placed against the wall is used to position oocytes in front of tools like injector, aspiration pipe, sensor, etc.

To test our system and its feasibility, we did some preliminary automatic open loop micromanipulation. Figure ?? presents a micro-object (200 $\mu$ m diameter sphere) having backward and forward motion. This micromanipulation example proves the feasibility of our concept. Without visual feedback, the speed system is limited by the inertia of the permanent magnet. In case of a visual control, the speed sytem is function of the camera and visual treatment time constants.

To optimize this principle, a complete modelling of the link between the magnet trajectory and micropusher trajectory is needed. Moreover to perform cell displacements, the micropusher trajectory must be controlled and the study of the automatic control need a complete dynamic model too. The behaviour modelling is presented in the following.

\section{Behaviour Modelling}

The modelling aims are to propose a complete model of the dynamic behaviour of the micropusher in function of the magnetic source movement where parameters are the shape of the micropusher, and the shape and the nature (permanent magnet, micro-coils) of the magnetic source.

Position between micropusher and magnet, presented in figure ??, is defined by two points: Center $O$ of the magnet top plan and contact point $I$ between the micropusher and the glass wall. The position of the micropusher in a inertial reference frame $R_{m}:\left(O_{m}, \vec{x}, \vec{y}, \vec{z}\right)$ is defined by $x_{m}, y_{m}$, and $z_{m}$. Relative positions between the point $O$ and the point $I$ is defined as $O I_{x}, O I_{y}$ and $O I_{z}$. Micropusher orientation is defined by two angles: $\alpha$ and $\beta$ defined in figure ??. Angle $\alpha$ enables movements like step 2 in figure 1 . Angle $\beta$ is useful for accurate positioning [4]. The micropusher is modelled by a plan surface $S$, with a normal $\vec{n}$ and a thickness $e$. Micro-pusher reference frame is defined by vectors: $\vec{n}, \vec{b}$ and $\vec{c}$ described in figure ??.
The model describes the behaviour of a ferromagnetic particle (whatever its plan shape is) into a magnetic field (whatever its source is). Consequently the model outputs are the position and the orientation of the micropusher, the inputs are the position of the magnetic source and the parameters are magnetic micropusher properties and the magnetic field $\overrightarrow{B_{0}}$ induced by the permanent magnet.

The model is composed of several blocks (see in figure ??) where each block represents one calculation step. First the finite element model "Flux3D ${ }^{\circledR}$ " is used to obtain magnetic field $\overrightarrow{B_{0}}$ according to the magnetic source shape and properties. The second step consists in the calculation of the magnetization $\vec{M}$ of the micropusher. Magnetic torque $\overrightarrow{\Gamma_{m, I}}$ in point $I$ and magnetic force $\overrightarrow{F_{m}}$ applied by the magnet on the micropusher are determined in function of the relative position $\overrightarrow{O I}$ between the micropusher and the magnet. These first steps are performed off-line and efforts $\left(\overrightarrow{F_{m}}, \overrightarrow{\Gamma_{m, I}}\right)$ are stored in a large numerical matrix.

The last step is the determination of the micropusher position $\left(x_{m}, y_{m}\right.$ and $\left.z_{m}\right)$ and orientation $(\alpha$ and $\beta)$ behaviour using the efforts stored. Each block principle is detailed in the following.

\section{A. Magnetic Block}

The magnetic block output is the micropusher internal magnetization $\vec{M}$ according to the micropusher geometrical characteristic and magnetic field $\overrightarrow{B_{0}}$. To build this function, two hypotheses are assumed: The micropusher is a flat surface $S$; the magnetization reached the saturation $M_{\text {sat }}$ :

$$
M_{\text {sat }}=\|\vec{M}\|=5.1 \times 10^{5} \mathrm{~A} . \mathrm{m}^{-1}
$$

Magnetization orientation can be calculated by computed the continuity relation of the magnetic field on the surface $S$ as described in [5].

\section{B. Strain Block}

To present the mechanical action applied by the magnetic source on the micropusher, a small surface $d S$ of the flat micropusher around the point $P$ is considered. Classical magnetic efforts applied on this element are:

- An elementary surface magnetic force $\overrightarrow{d F_{m}}$ :

$$
\overrightarrow{d F_{m}}=\vec{\nabla}\left(\vec{M} \cdot \overrightarrow{B_{0}}\right) \cdot d S
$$

- An elementary surface magnetic torque $\overrightarrow{d \Gamma_{m}}$ :

$$
\overrightarrow{d \Gamma_{m}}=\vec{M} \wedge \overrightarrow{B_{0}} \cdot d S
$$

The complete magnetic effort applied on the pusher in the point $I$ is thus:

$$
\begin{aligned}
& \overrightarrow{F_{m}}=e \times \int_{S} \overrightarrow{d F_{m}} \\
& \overrightarrow{\Gamma_{m, I}}=e \cdot \int_{S} \overrightarrow{d \Gamma_{m}}+e \cdot \int_{S} \overrightarrow{P I} \wedge \overrightarrow{d F_{m}}
\end{aligned}
$$

where $e$ is the thickness of the flat micropusher. 


\section{Dynamic Block}

The dynamic behaviour is defined by the equilibrium between efforts and dynamic terms in point $I$ :

$$
\begin{aligned}
\overrightarrow{F_{f}}+\overrightarrow{F_{m}} & =m \vec{\Gamma} \\
\overrightarrow{\Gamma_{m, I}} & =J \cdot \vec{\gamma}
\end{aligned}
$$

where $\overrightarrow{F_{f}}$ is the action of the glass wall on the micropusher applied in point $I, m$ the micropusher mass, $\vec{\Gamma}$ its acceleration, $J$ its angular inertia, and $\vec{\gamma}$ the angular acceleration.

The dynamic behaviour in translation is a function of the magnetic force $\overrightarrow{F_{m}}$ and of friction conditions $\overrightarrow{F_{f}}$. The angular behaviour is only a function of magnetic effects $\overrightarrow{\Gamma_{m, I}}$.

The dynamic behaviour is the superposition of three elementary dynamic behaviours described as:

- The angular behaviour $\alpha$ with axe $(I, \vec{z})$;

- The angular behaviour $\beta$ with axe $(I, \vec{b})$;

- The translation behaviour $\left(x_{m}, y_{m}\right)$.

By computation of the dynamic equations (6), (7) the time constants of these behaviours are determined. These values is presented in table I.

\begin{tabular}{|c|c|}
\hline $\begin{array}{c}\text { Elementary Dynamic } \\
\text { Behaviours }\end{array}$ & Time Constant \\
\hline Rotation $\alpha$ & $45 \times 10^{-6} \mathrm{~s}$ \\
\hline Rotation $\beta$ & $37 \times 10^{-6} \mathrm{~s}$ \\
\hline Translation $\left(x_{m}, y_{m}\right)$ & $930 \times 10^{-6} \mathrm{~s}$ \\
\hline \multicolumn{3}{|c}{ TABLE I } \\
\hline
\end{tabular}

Time Constant of the Three Elementary Dynamic BEHAVIOUR

The time constants of the angular behaviour are negligible compared to the translation time constant. Moreover, these time constants are very small compared to the time constant of the controller (around $1 \mathrm{~ms}$ ) or compared to the lower time constant in our device: the time constant of the camera $(20 \mathrm{~ms})$ used to measure the micropusher position. Consequently the general dynamic behaviour can be considered as a dynamic behaviour in translation and a quasi-static behaviour in rotation. Both behaviours are detailed in the following sections

1) Angular Behaviour: At each time $t$, we consider that the micro-pusher is reaching its angular static equilibrium. The angular behaviour presented in (7) is consequently reduced as:

$$
\overrightarrow{0}=\overrightarrow{\Gamma_{m, I}}=e \cdot \int_{S} \overrightarrow{d \Gamma_{m}}+e \cdot \int_{S} \overrightarrow{P I} \wedge \overrightarrow{d F_{m}}
$$

The angular position is only done by the equilibrium between both terms of the magnetic torque $\overrightarrow{\Gamma_{m, I}}$. Consequently, its calculation in function of the relative position $\overrightarrow{O I}$ can be determined in pre-processing. Thus, the dynamic block can be presented as described in figure ??. Angular positions are defined in function of the torque $\overrightarrow{\Gamma_{m, I}}$ and are stored in a large numerical matrix. Translation behaviour is simulated in function of the position of the magnet and the stored magnetic forces. At each time $t$, the angle of the micropusher is calculated by interpolation of the stored angular positions.
2) Translation behaviour: The micropusher translation behaviour (6) is a function of the friction force applied by the glass vertical wall. This force $\overrightarrow{F_{f}}$ can be divided into:

- A normal component $\overrightarrow{F_{f N}} \cdot \vec{z}$ perpendicular to the contact surface which is in equilibrium with the normal magnetic force:

$$
\overrightarrow{F_{f N}} \cdot \vec{z}=-\overrightarrow{F_{m}} \cdot \vec{z}
$$

- A tangential component $\overrightarrow{F_{f T}}$ sum of both components $F_{f T x} \cdot \vec{x}$ and $F_{f T y} \cdot \vec{y}$.

To describe the translation behaviour of the micro-pusher, two cases are studied:

- The point $I$ is immobile and the movement of the micropusher is only a rotation;

- The point $I$ moves, and the micropusher is in translation on the vertical glass slide.

When the micropusher contact point $I$ is immobile, the friction tangential component $\overrightarrow{F_{f T}}$ is lower than the product of the static friction coefficient $f_{s}$ and the normal component $\overrightarrow{F_{f N}}$ :

$$
\left\|\overrightarrow{F_{f T}}\right\|<f_{s} .\left\|\overrightarrow{F_{f N}}\right\|
$$

In this case, the tangential component of the friction force and the tangential magnetic force stay in equilibrium:

$$
\left\{\begin{array}{l}
\overrightarrow{F_{f T}} \cdot \vec{x}+\overrightarrow{F_{m}} \cdot \vec{x}=0 \\
\overrightarrow{F_{f T}} \cdot \vec{y}+\overrightarrow{F_{m}} \cdot \vec{y}=0
\end{array}\right.
$$

When the norm of the tangential component reaches the product $f_{s} .\left\|\overrightarrow{F_{f N}}\right\|$, the contact point $I$ moves:

$$
\left\|\overrightarrow{F_{f T}}\right\|=f_{s} \cdot\left\|\overrightarrow{F_{f N}}\right\| \Rightarrow \overrightarrow{V_{I}} \neq 0
$$

where $\overrightarrow{V_{I}}$ is the velocity of the point $I$.

When the micropusher is in movement with a velocity $\overrightarrow{V_{I}}$, the modulus of the friction tangential force is the product of the dynamic friction coefficient $f_{d}\left(f_{d} \leq f_{s}\right)$ and the normal component $\left\|\overrightarrow{F_{f N}}\right\|$ :

$$
\overrightarrow{F_{f T}}=-f_{d} \times\left\|\overrightarrow{F_{f N}}\right\| \cdot \overrightarrow{n_{v}}
$$

where $\overrightarrow{n_{v}}$ is the unit vector of the velocity $\overrightarrow{V_{I}}$.

The translation dynamic behaviour of the micropusher is defined by the projection of the equation (6) in the wall plan $(\vec{x}, \vec{y})$, thus:

$$
\begin{aligned}
& \left\{\begin{array}{l}
\overrightarrow{F_{m}} \cdot \vec{x}+\overrightarrow{F_{f T}} \cdot \vec{x}=m \ddot{x}_{m} \\
\overrightarrow{F_{m}} \cdot \vec{y}+\overrightarrow{F_{f T}} \cdot \vec{y}=m \ddot{y}_{m}
\end{array}\right. \\
& \left\{\begin{array}{l}
\overrightarrow{F_{m}} \cdot \vec{x}-f_{d} \cdot\left\|\overrightarrow{F_{f N}}\right\| \cdot \overrightarrow{n_{v}} \cdot \vec{x}=m \ddot{x}_{m} \\
\overrightarrow{F_{m}} \cdot \vec{y}-f_{d} \cdot\left\|\overrightarrow{F_{f N}}\right\| \cdot \overrightarrow{n_{v}} \cdot \vec{y}=m \ddot{y}_{m}
\end{array}\right. \\
& \left\{\begin{aligned}
x_{m}= & \frac{1}{m} \iint\left(\overrightarrow{F_{m}} \cdot \vec{x}-f_{d} \cdot\left\|\overrightarrow{F_{f N}}\right\| \cdot \overrightarrow{n_{v}} \cdot \vec{x}\right) \cdot d^{2} t \\
& +\dot{x}_{m}(0) t+x_{m}(0) \\
y_{m}= & \frac{1}{m} \iint\left(\overrightarrow{F_{m}} \cdot \vec{y}-f_{d} \cdot\left\|\overrightarrow{F_{f N}}\right\| \cdot \overrightarrow{n_{v}} \cdot \vec{y}\right) \cdot d^{2} t \\
& +\dot{y}_{m}(0) t+y_{m}(0)
\end{aligned}\right.
\end{aligned}
$$


The equations (11) and (16) define respectively the behaviour of the micro-pusher when the point $I$ is immobile and when it is in movement. The equation (12) defines the transition condition.

Moreover when $f_{d} \neq f_{s}$, the stick-slip motion appears as presented in [13]. When the magnet moves, the micropusher stays first immobile until the magnetic tangential force reaches the static friction condition (equation (12)). Thus, the micro-pusher is moving in function of the dynamic behaviour (equation 16), and a large acceleration is applied to the micropusher until it reaches a new static equilibrium position.

This phenomenon increases the difficulties to control the final position of the micro-pusher or the pushed object position. Some original control strategies are proposed in [4] to overcome stick-slip.

\section{Experimental and Simulation Results}

The quasi-static angular behaviour and planar trajectory of the micropusher was compared with experimental measurements.

\section{A. Angular Behaviour}

The first experimental validation deals with the equilibrium angle of the micropusher (angle $\beta$ in figure ??) versus the micropusher size and the relative position $\overrightarrow{O I}$. These tests allows to compare computed and measured values. The calculated angles $\alpha$ and $\beta$ are determined by the numerical resolution of equilibrium equation (8).

As shown by equation (8), the angular position is done by the equilibrium of two physical phenomena: The magnetic torques $\overrightarrow{d \Gamma_{m}}$ and the torques in $I$ induced by the magnetic force $\overrightarrow{d F_{m}}$ :

$$
\int_{S} \overrightarrow{d \Gamma_{m}}=-\int_{S} \overrightarrow{P I} \wedge \overrightarrow{d F_{m}}
$$

These two strains induce two different micropusher behaviours:

The magnetic torques $\overrightarrow{d \Gamma_{m}}$ consequence are the alignment of the micropusher on the external magnetic field $\overrightarrow{B_{0}}$ (see equation 3). On the opposite, the torque induced by the magnetic force $\overrightarrow{P I} \wedge \overrightarrow{d F_{m}}$ makes the micropusher lying flat on the plan.

In fact the first term is function of $l^{3}$ and the second is function of $l^{4}$ where $l$ is the micropusher characteristic size. Consequently, smaller is the micropusher and more important is the magnetic torque $\overrightarrow{d \Gamma_{m}}$ compared to the magnetic force torque $\overrightarrow{P I} \wedge \overrightarrow{d F_{m}}$. Physically, smaller is the micropusher and closer to the magnetic field line is its orientation.

Simulations and experimental measurements is presented for the following case: $-500 \mu m<O I_{x}<+500 \mu m$; $\mathrm{OI}_{y}=0$; $\mathrm{OI}_{z}=200 \mu \mathrm{m}$.

Micropusher orientation is measured thanks to visual treatments of lateral views. The experimental measurements and simulations results are presented in figure ??. The micropusher is not aligned on magnetic field $\overrightarrow{B_{0}}$. Actually, magnetic torque is not prominent compared to the magnetic force torque. In the opposite, the micropusher is also not lying the glass slide because magnetic force torque is not prominent compared to the magnetic torque. Both phenomena have the same value order and the micropusher orientation is defined between this two extremum position. Experimental measurements and our model are similar.

\section{B. Dynamic Comparison}

This section presents the comparison between the computed trajectories and experimental measurements. The simulation of the pusher trajectory is based on the magnetic force calculation (4) computed for each relative position $\overrightarrow{O I}$ in preprocessing. Simulated trajectory is obtained by resolution of the equations (11), (12) and (16) in Matlab Simulink. The experimental position of the micro-pusher is measured by a CCD Camera and a visual treatment.

The example presented in figure ?? is obtained with a square trajectory of the magnet. A good correlation between both results is observed, and trajectories are relatively near.

Moreover, the model allows to computed trajectories in function of the magnetic source shape. We presented the differences observed between two permanent magnets:

- A cylindrical magnet $1.6 \mathrm{~mm}$ in diameter, $0.74 \mathrm{~mm}$ in thickness and axis $z$;

- A square magnet $\left(1.6 \times 1.6 \times 0.74 \mathrm{~mm}^{3}\right)$.

Both magnets have a remanent magnetic field oriented along the $z$ axis. Both trajectories are presented in figure ??.

The second geometry induces a modification of the magnetic field $\overrightarrow{B_{0}}$ simulated by the software Flux 3D. This modification induces a new dynamic behaviour and a modification of the micro-pusher trajectory.

The model build is designed to be generic and is able to determine the dynamic behaviour of the micropusher in function of the magnet movement for different magnet shapes, and micropusher shapes and properties. Moreover, as the model is only based on the magnet field induced by a magnetic source it can be generalized to micro-coils.

\section{Conclusion}

A new approach to manipulate cell by using a small micropusher was described. The submerged ferromagnetic micropusher follows the movement of a magnet located in the air. We show that the manipulation of oocyte is possible with this device. A complete generic model of the micropusher trajectory is proposed. It allows to predict the micropusher movement in function of the magnet position according to the micropusher shape and properties and magnetic field induced by the magnet. This generic model can be generalized to other magnetic source like micro-coils. This simulation tool will be the base of further works on the optimization of the micropusher architecture and on the development of automatic pushing tasks.

\section{ACKNOWLEDGMENTS}

This research was supported by the LAB (Laboratory of Automation of Besançon - France). The authors thank Prof. C. Roux (research group on "genetic and reproduction", Besançon) for collaboration on human oocytes micromanipulations. The authors thank the LCEP-Besançon for the fabrication of the micropusher glass sample support. 


\section{REFERENCES}

[1] X. Li, G. Zong, S. Bi, and W. Zhao, "Automatic micromanipulating system for biological applications with visual servo control," Journal of Micromechatronics, vol. 1, no. 4, pp. 34563, 2002.

[2] Y. Sun, M. A. Greminger, and B. J. Nelson, "Investigating protein structure with a microrobotic system," in Proceedings of the 2004 IEEE ICRA, New Orleans, USA, April 2004, pp. $2854-59$.

[3] B. K. Kim, D. H. Kim, J. Park, Y. Kim, S. J. Kwon, H. Kang, and S. H. Jung, "Autonomous biomanipulation factory for manipulating individual embryo cells," in Proceedings of the Workshop Microrobotics for biomanipulation, IROSO3, Las Vegas, USA, Oct 2003, pp. 74-88.

[4] M. Gauthier and E. Piat, "Control of a particular micro-macro positionning system applied to cell microdisplacement," IEEE Transactions on Automation Science and Engineering, vol. 3, no. 3 , pp. 264-271, july 2006 .

[5] M. Dauge, M. Gauthier, and E. Piat, "Modelling of a $2 \mathrm{~d}$ magnetic cell transport system," in proc. of the IEEE Int. Conf. on Intelligent Robots and Systems, August 2005, pp. 4098-4103.

[6] O. Cugat, J. Delamare, and G. Reyne, "Magnetic microactuators and systems (magmas)," IEEE Transactions on Magnetics, vol. 39, no. 6, pp. 3607-12, nov. 2003.

[7] A. Hatch, A. E. Kamholz, G. Holman, P. Yager, and K. F. Böhringer, "A ferrofluidic magnetic micropump," Journal of Microelectromechanical Systems, vol. 10, no. 2, pp. 215-221, June 2001.

[8] L. K. Lagorce, O. Brand, and M. Allen, "Magnetic microatuator based on polymer magnets," Journal of micromechanical systems, vol. 8, no. 1, pp. 3-14, 1999.

[9] K. B. Yesin, K. Vollmers, and B. J. Nelson, "Analysis and design of wireless magnetically guided microrobots in body fluids." in Proceedings of the 2004 IEEE ICRA, New Orleans, USA, April 2004, pp. 1333-38.

[10] F. Arai, H. Maruyama, T. S aadn A. Ichikawa, and T. Fukuda, "Non-contact manipulation of biological cells," in Proceedings of the Workshop Microrobtics for biomanipulation, IROS03, Las Vegas, USA, Oct 2003, pp. 50-60.

[11] M. Gauthier and E. Piat, "Microfabrication and scale effect studies for a magnetic micromanipulation system," in Proc. of the IEEE International Conference on Intelligent Robots and Systems - IROS02, vol. 2, Lausanne - Switzerland, 30 sept - 4 Oct 2002 , pp. $1754-59$

[12] A. Ichikawa, F. Arai, H. Maruyama, T. Fukuda, and T. Katsuragi, "Single cell trap on a chip using in-situ microfabrication with photo-crosslinkable resin and thermal gelation," in Proceedings of the 2004 IEEE ICRA, New Orleans, USA, April 2004, pp. 2848-53.

[13] M. Gauthier and E. Piat, "An electromagnetic micromanipulation system for single cell manipulation," Journal of Micromechatronics, vol. 2, no. 2, pp. 87-119, Feb 2004. 